\title{
Nova espécie de Novamundoniscus Schultz (Isopoda, Oniscidea, Dubioniscidae) para o Rio Grande do Sul, Brasil ${ }^{1}$
}

\author{
Elis Regina de Carvalho Lopes ${ }^{2} \&$ Paula Beatriz Araujo ${ }^{3}$
}

${ }^{1}$ Contribuição número 422 do Departamento de Zoologia, Instituto de Biociências, Universidade Federal do Rio Grande do Sul.

2 Laboratório de Crustáceos Decápodos, Programa de Pós-Graduação em Oceanografia Biológica, Departamento de Oceanografia, Fundação Universidade Federal do Rio Grande. Avenida Itália, Km 8, Caixa Postal 474, 96201 -900 Rio Grande, Rio Grande do Sul, Brasil.

3) Programa de Pós-Graduação em Biologia Animal, Departamento de Zoologia, Instituto de Biociências, Universidade Federal do Rio Grande do Sul. Avenida Bento Gonçalves 9500, prédio 43435, 91501-970 Porto Alegre, Rio Grande do Sul, Brasil.

\begin{abstract}
New species of Novamundoniscus Schultz (Isopoda, Oniscidea, Dubioniscidae) from Rio Grande do Sul, Brazil. From material collected in the northeast of Rio Grande do Sul State, one new species of Novamundoniscus Schultz, 1995 is described.
\end{abstract}

KEY WORDS. Crustacea, taxonomy, terrestrial isopods.

A família Dubioniscidae foi criada por Schultz (1995) para incluir, além dos gêneros já conhecidos Dubioniscus Vandel, 1963, Calycuoniscus Collinge, 1915 e Phalloniscus Budde-Lund, 1908, o gênero Novamundoniscus Schultz, 1995, que foi criado para abrigar as espécies americanas, previamente incluídas em Phalloniscus. Segundo o autor, Novamundoniscus possui uma combinação de caracteres peculiares, a qual nenhuma espécie de Phalloniscus da Nova Zelândia ou Europa a possui, sendo esta: abdome tão largo quanto o tórax com neopleurons 3-5 bem desenvolvidos; processo molar da mandíbula composto; exópodo da maxílula com dentes regulares; endito do maxilípodo simples com uma seta, artículo apical com setas distintas, não com tufo de setas, setas distintas em dois grupos na margem interna do segundo artículo do palpo, artículo basal com pelo menos duas setas longas; pleópodos sem área respiratória.

De acordo com Lemos de Castro (1960), o gênero Phalloniscus foi instituído sem apresentação de diagnose, para abrigar espécies da Nova Zelândia, anteriormente incluídas em Oniscus Linnaeus, 1755. VANDEL (1952) discutiu esta validade e apresentou a diagnose de Phalloniscus, considerando sua distribuição na América, para Venezuela, Bolívia, Trindad e Guianas. Mais tarde, VANDEL (1962) apresentou uma revisão de todas as espécies do gênero, para uma possível ordenação, talvez em diferentes gêneros afins. Schultz (1995) quando da criação de Dubioniscidae e da inclusão das espécies americanas de Phalloniscus em Novamundoniscus, sem qualquer comentário ou esclarecimento, não incluiu ao novo gênero todas as espécies americanas de Phalloniscus. A análise do material tipo de Phalloniscus meridionalis Araujo \& Buckup, 1994, permitiu a inclusão desta espécie em Novamundoniscus. No Brasil, até o momento, são conhecidas seis espécies desse gênero registradas nos Estados do Rio de Janeiro, Minas Gerais, Amazonas, Pará (Souza-Kury 1998), Santa Catarina e Rio Grande do Sul (Araujo \& BUCKUP 1994). Em coletas realizadas na região nordeste do Rio Grande do Sul, em locais caracterizados por matas de restinga e florestas de araucárias, foi encontrada uma nova espécie do gênero, descrita aqui como Novamundoniscus gracilis sp. nov.

O material examinado encontra-se depositado no $\mathrm{Mu}$ seu Nacional, Rio de Janeiro (MNRJ) e Departamento de Zoologia da Universidade Federal do Rio Grande do Sul, Porto Alegre (UFRGS).

\section{Novamundoniscus gracilis sp. nov. Figs $1-20$}

Descrição. Antena com o segundo artículo do flagelo maior que o primeiro e do que o terceiro, sendo este último, muito menor que os demais, maxílula com $4+4$ dentes inteiros, dimorfismo sexual nos pereiópodos 1 e 2 , os quais apresentam um conjunto de setas bifurcadas no mero e no carpo e pereiópodo 7, que apresenta longas e finas setas no ísquio nos machos. $4,2 \mathrm{~mm}$

Comprimento. Maior macho, 3,6; maior fêmea ovígera,

Revista Brasileira de Zoologia 20 (4): 611-614, dezembro 2003 
Coloração. Castanha com regiões despigmentadas alongadas nas margens laterais dos pereionitos; pereionitos IIII quase totalmente despigmentados na região mediana. Antena, em geral, com o pedúnculo despigmentado nos três primeiros artículos e parte do quarto; flagelo antenar com coloração castanha uniforme. Urópodos com coloração castanha uniforme (Fig. 1).

Cefalotórax. Olhos compostos com 15 omatídeos.

Pereion. Superfície corporal lisa, coberta por setas escamosas em forma de leque (Fig. 2), sem poros glandulares nos pereionitos. Nódulos laterais dispostos um em cada lado do pereionito 1-7; posição das coordenadas b/c e d/c como na figura 3.

Pleon. Estreitando-se a partir do limite posterior do pereion, com setas escamosas semelhantes às do pereion; epímeros 3-5 desenvolvidos, com as extremidades dirigidas para trás. Telso triangular curto, com as margens laterais levemente côncavas e ápice arredondado, não ultrapassando o protopodito dos urópodos. Inserção dos endópodos e exópodos dos urópodos em altura diferente; endópodos dos urópodos atingindo quase a metade dos exópodos (Fig. 4).

Apêndices. Antênula: com o artículo distal portando 4-5 estetascos; não há estetascos apicais (Fig. 6). Antena: quando distendida para trás, ultrapassa a margem posterior do terceiro pereionito; o segundo artículo do flagelo é o mais longo e o terceiro muito menor que os demais (Fig. 5). Mandíbula: direita com dois e esquerda com três penicílios no processo incisor; processo molar dicotomizado (Figs 7 e 8). Maxílula: endito com dois penicílios apicais; exito com $4+4$ dentes inteiros (Fig. 9). Maxila:. lobo externo subretangular (Fig. 10). Maxilípodo: com placas escamosas; endito quadrangular com uma seta robusta, margem posterior levemente sinuosa, com dois dentículos; palpo com duas setas longas e robustas no artículo basal, artículo apical terminando em setas distintas e longas (Fig. 11). Pereiópodos: pereiópodos 1 e 2 do macho com o carpo apresentando uma fileira de setas bifurcadas (Fig. 13); pereiópodo 7 com tufo de setas finas e longas no ísquio (Fig. 14). Pleópodos: exópodo do pleópodo 1 arredondado, alongando-se distalmente, formando uma pequena projeção no ápice posterior (Fig. 12); endópodo do pleópodo 1 alongado, com setas pequenas e curtas na extremidade distal (Fig. 15). Pleópodo 2 com exópodo alongado, com uma seta robusta na margem externa e margem interna proximal de contorno sinuoso (Fig. 16); endópodo longo, atingindo a margem posterior do exópodo do pleópodo 5, afilando-se a partir do terço distal (Fig. 17). Exópodos 3-5 apresentando entre 2-5 setas na margem lateral externa (Fig. 18-20).

Holótipo macho. Brasil, Rio Grande do Sul: Torres (Itapeva, $29^{\circ} 38^{\prime} \mathrm{S} 49^{\circ} 78^{\prime} \mathrm{W}$, em serrapilheira de vegetação de restinga), 1 macho, 9.XII.1998, E.R.C. Lopes leg. (MNRJ, 18827).

Parátipos. BRASIL, Rio Grande do Sul: São Francisco de Paula (Rota do Sol, $29^{\circ} 17^{\prime} \mathrm{S} 50^{\circ} 15 \mathrm{~W}$, em serapilheira de Floresta de Araucária), 1 macho, 1 fêmea, 19.IX.1999, E.R.C. Lopes, P.B. Araujo e G. Bond-Buckup leg. (UFRGS, 3618P); São Francisco de Paula (Rota do Sol, $29^{\circ} 17^{\prime} \mathrm{S} 50^{\circ} 16^{\prime} \mathrm{W}$, em serapilheira de Floresta de Araucária), 1 fêmea, 19.IX.1999, E.R.C. Lopes leg. (UFRGS, 3619P); São Francisco de Paula (Tainhas, $29^{\circ} 32^{\prime}$ 'S $50^{\circ} 41^{\prime} \mathrm{W}$, em serapilheira de Floresta de Araucária), 1 macho, 26.XI.1998, E.R.C. Lopes, A.O. Almeida e L.R. Bernardi leg. (UFRGS, 3620P); Torres (Itapeva 29 $38^{\prime} \mathrm{S} 49^{\circ} 78^{\prime} \mathrm{W}$, em serapilheira de vegetação de restinga), 2 machos, 09.XII.1998,
E.R.C. Lopes leg. (UFRGS, 3621P); Torres (Itapeva $29^{\circ} 23^{\prime}$ S $49^{\circ} 45^{\prime} \mathrm{W}$, em serapilheira de vegetação de dunas), 4 machos, 7 fêmeas, 4 fêmeas ovígeras, 10.IX.1998, E.R.C. Lopes, P.B. Araujo e G. Bond-Buckup leg. (UFRGS, 3622P); São Francisco de Paula, Tainhas, $29^{\circ} 37^{\prime} \mathrm{S} 50^{\circ} 43^{\prime} \mathrm{W}$, em serapilheira de Floresta de Araucária), 2 machos, 2 fêmeas ovígeras, 06.XI.1998, E.R.C. Lopes leg. (UFRGS, 3623P); São Francisco de Paula $\left(29^{\circ} 42^{\prime}\right.$ S $50^{\circ} 48^{\prime} \mathrm{W}$, em serapilheira de Floresta de Araucária), 1 fêmea, 06.XI.1998, E.R.C. Lopes, M.M. Gralla e T.S. Castro leg. (UFRGS, $3624 \mathrm{P})$; Arroio do Sal (29 $27^{\prime} \mathrm{S} 49^{\circ} 50^{\prime} \mathrm{W}$, em serapilheira de vegetação de restinga), 1 macho, 03.III.1999, E.R.C. Lopes leg.

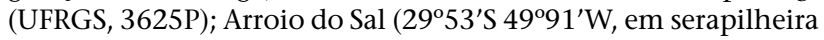
de vegetação de restinga), 2 machos, 1 fêmea, 3 fêmeas ovígeras), 09.XII.1998, E.R.C. Lopes e A.A.P. Bueno leg. (UFRGS, $3626 \mathrm{P})$; Arroio do Sal (29 $56^{\prime} \mathrm{S} 49^{\circ} 93^{\prime} \mathrm{W}$, em serapilheira de vegetação de restinga), 11 machos, 13 fêmeas, 07 fêmeas ovígeras), 09.XII.1998, E.R.C. Lopes e A.A.P. Bueno leg. (UFRGS, 3627P); Arroio do Sal (29 $56^{\prime} \mathrm{S} 49^{\circ} 93^{\prime} \mathrm{W}$, em serapilheira de vegetação de restinga), 4 machos, 1 fêmeas ovígeras, 25.IX.1999, E.R.C. Lopes leg. (UFRGS, 3628P); Arroio do Sal (29 $33^{\prime} \mathrm{S} 49^{\circ} 55^{\prime} \mathrm{W}$, em serapilheira de vegetação de restinga), 1 macho, 1 fêmea ovígera, 25.IX.1999, E.R.C. Lopes leg. (UFRGS, 3629P); Capão da Canoa

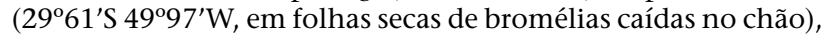
1 macho, 1 fêmeas ovígeras, 10.IX.1998, E.R.C. Lopes, P.B. Araujo e G. Bond-Buckup leg. (UFRGS, 3630P); Capão da Canoa $\left(29^{\circ} 63^{\prime} \mathrm{S} 49^{\circ} 96^{\prime} \mathrm{W}\right.$, em serapilheira de vegetação de restinga), 10 machos, 8 fêmeas, 16 fêmeas ovígeras, 10.IX.1998, E.R.C. Lopes, P.B. Araujo e G. Bond-Buckup leg. (UFRGS, 3631P); Capão da Canoa $\left(29^{\circ} 49^{\prime} \mathrm{S} 50^{\circ} 04^{\prime} \mathrm{W}\right.$, em serapilheira de vegetação de restinga), 4 machos, 7 fêmeas, 7 fêmeas ovígeras, 07.IV.2003, E.R.C. Lopes, P.B. Araujo e G. Bond-Buckup leg. (UFRGS, 3632P).

Habitats. A espécie foi freqüentemente coletada na Região Costeira, junto à Mata de Restinga, em uma faixa de 0-100 metros de altitude, bem como em alguns pontos isolados do Planalto Rio-Grandense, junto à Floresta de Araucária, numa faixa de altitude entre 900 e $1000 \mathrm{~m}$. Em ambos os ambientes, os exemplares de Novamundoniscus gracilis sp.nov., foram coletados junto à vegetação nativa ou pouco alterada pela atividade humana.

Discussão Taxonômica. Novamundoniscus gracilis sp. nov. assemelha-se a Novamundoniscus meridionalis (Araujo \& Buckup, 1994) pelo número de penicílios no processo incisor das mandíbulas direita e esquerda, pela presença de dois dentículos na margem superior do endito do maxilípodo e pela forma do exópodo do pleópodo 1 do macho. Entretanto, diferencia-se desta por apresentar olhos com 15 omatídeos (10-12 em $N$. meridionalis), possuir $4+4$ dentes inteiros na maxílula $(4+4$ bífidos em N. meridionalis), por não apresentar estetascos apicais na antênula e pelo tamanho dos artículos do flagelo da antena. Possui semelhança também com Novamundoniscus singularis (Lemos de Castro, 1967) pelo número de omatídeos, mas diferencia-se por possuir $4+4$ dentes inteiros na maxílula $(4+4$ bífidos em $N$. singularis) e pela forma do pleópodo 1 do macho (exópodo trapezoidal e endópodo portando esporão na margem interna, próximo à extermidade distal em N. singularis).

\section{AGRADECIMENTOS}

Aos colegas do DZ-UFRGS que auxiliaram nos trabalhos de campo, a CAPES pela concessão de bolsa de mestrado à primeira autora e ao PPG Biologia Animal pela concessão do auxílio financeiro para a realização dos trabalhos de coleta. 


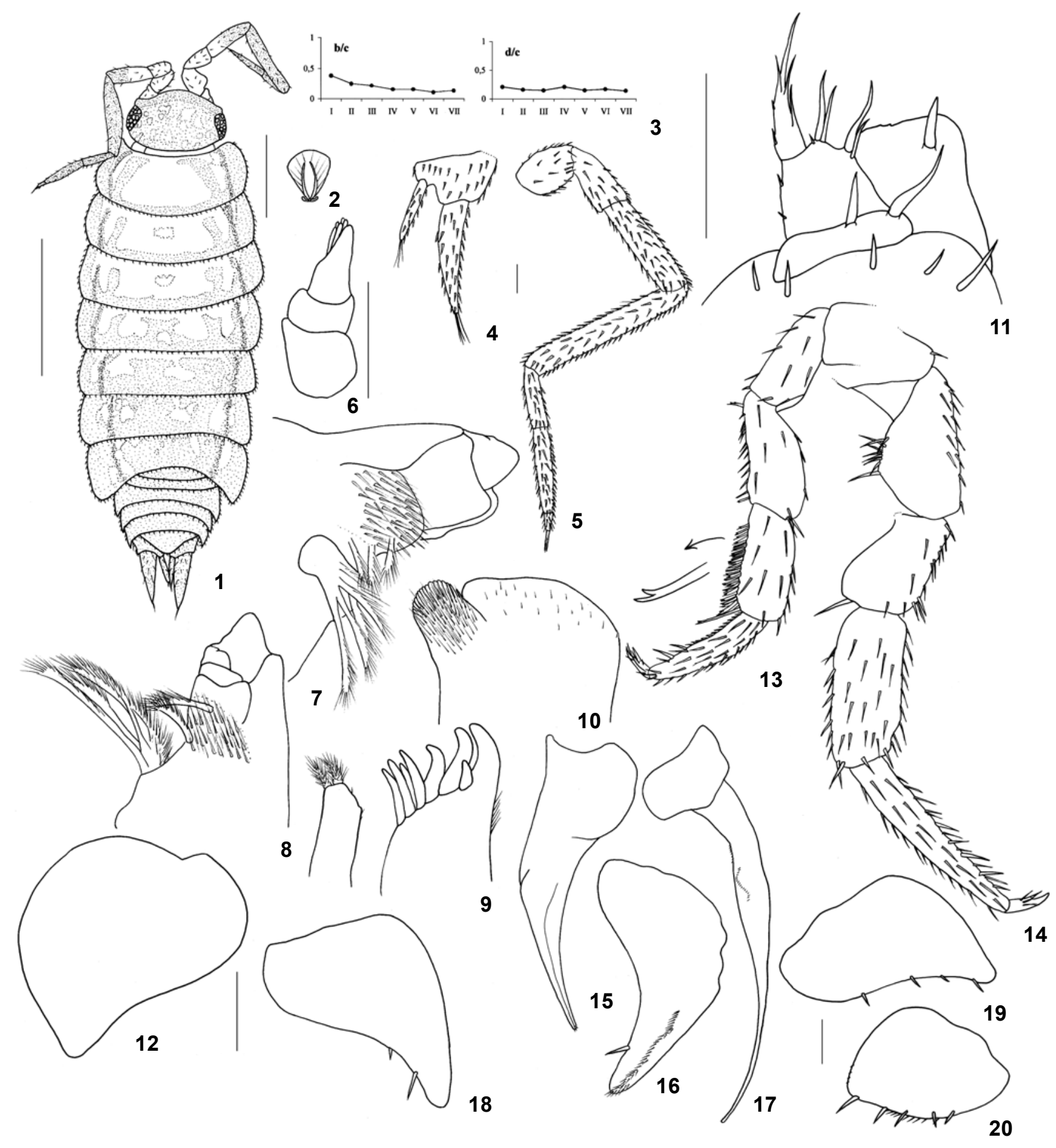

Figuras 1-20. Novamundoniscus gracilis sp. nov. macho: (1) holótipo, vista dorsal; (2) seta escamosa; (3) coordenadas b/c e d/c dos nódulos laterais; (4) urópodo; (5) antena; (6) antênula; (7) mandíbula esquerda; (8) mandíbula direita; (9) maxílula; (10) maxila; (11) maxilípodo; (12) exópodo 1; (13) pereiópodo 1; (14) pereiópodo 7; (15) endópodo 1; (16) exópodo 2; (17) endópodo 2; (18) exópodo 3; (19) exópodo 4; (20) exópodo 5. Escalas: Fig. 1: 1mm; Fig. 2: 0,05mm; Figs 4-20: 0,1 mm. 


\section{REFERÊNCIAS BIBLIOGRÁFICAS}

Araujo, P.B. \& L. Buckup. 1994. Two new species of terrestrial Isopoda from southern Brazil. Spixiana, München, 17 (3): 269-274.

Lemos de Castro, A. 1960. Sobre as espécies americanas de Phalloniscus Budde-Lund (Isopoda, Oniscoidea), com descrição de quatro espécies novas. Acta y trabajos del Primer Congreso Sudamericano de Zoologia, La Plata, 2: 203-211.

Schultz, G.A. 1995. Terrestrial isopod crustaceans (Oniscidea) from Paraguay with definition of a new family. Revue Suisse de Zoologie, Geneve, 102 (2): 387-424.

SouZA-KunY, L.A. 1998. Malacostraca-Peracarida. Isopoda. Oniscidea, p. 653-674. In: P.S. Young (Ed.). Catalogue of Crustacea of Brazil. Rio de Janeiro, Museu Nacional, 718p. VANDEL, A. 1952. Étude des Isopodes terrestres récoltés au Venezuela par lê Dr. G. Marcuzzi, suivie de considerations sur lê peuplemente du Continent de Gondwana. Memorie del Museo Civico di Storia Naturale di Verona, Verona, 3: 135-145.

. 1962. Isopodes Terrestres (Deuxieme partie). In: Faune de France. P. Lechevalier, Paris, 66: 417-931.

Recebido em 06.VI.2003; aceito em 17.X.2003.

Revista Brasileira de Zoologia 20 (4): 611-614, dezembro 2003 